\title{
HIP-20: Integration of Vehicle-HSM-Generated Credentials into Plug-and-Charge Infrastructure
}

\author{
Andreas Fuchs \\ andreas.fuchs@sit.fraunhofer.de \\ Fraunhofer SIT \\ Darmstadt, Germany
}

\author{
Dustin Kern \\ dustin.kern@sit.fraunhofer.de \\ Fraunhofer SIT \\ Darmstadt, Germany
}

\author{
Christoph Krauß $\beta^{*}$ \\ christoph.krauss@sit.fraunhofer.de \\ Fraunhofer SIT \\ Darmstadt, Germany
}

\author{
Maria Zhdanova \\ maria.zhdanova@sit.fraunhofer.de \\ Fraunhofer SIT \\ Darmstadt, Germany
}

\author{
Ronald Heddergott \\ ronald.heddergott@carmeq.com \\ Carmeq $\mathrm{GmbH}$ \\ Berlin, Germany
}

\begin{abstract}
Plug-and-Charge (PnC) standards such as ISO 15118-20 enable the charging of Electric Vehicles (EVs) with (nearly) no user intervention by storing authentication credentials directly in the vehicle. However, these credentials are generated in backend systems of vehicle manufacturers and charging service providers (called Mobility Operators (MOs)) making them vulnerable to attacks on corporate networks. In previous work, we proposed HIP, an ISO 15118 extension where credentials are generated in a Hardware Security Module (HSM) within the EV and the private parts of the credentials never leave the HSM. In this paper, we propose HIP-20, which improves HIP by enabling easy integration into existing backend infrastructures and processes. HIP-20 enables support of existing Certificate Authorities (CAs) and established processes such as using Certificate Signing Requests (CSRs), enables credential provisioning via channels outside of ISO 15118, and makes necessary adaptations required due to changes in the new edition ISO 15118-20. Our evaluation shows that HIP-20 significantly increases security and interoperability with existing processes and infrastructures.
\end{abstract}

\section{CCS CONCEPTS}

- Security and privacy $\rightarrow$ Hardware-based security protocols.

\section{KEYWORDS}

Automotive Security, ISO 15118, PKI, HSM, TPM

\section{ACM Reference Format:}

Andreas Fuchs, Dustin Kern, Christoph Krauß, Maria Zhdanova, and Ronald Heddergott. 2020. HIP-20: Integration of Vehicle-HSM-Generated Credentials into Plug-and-Charge Infrastructure. In Computer Science in Cars Symposium (CSCS '20), December 2, 2020, Feldkirchen, Germany. ACM, New York, NY, USA, 10 pages. https://doi.org/10.1145/3385958.3430483

*Also with Darmstadt University of Applied Sciences.

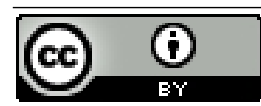

This work is licensed under a Creative Commons Attribution International 4.0 License CSCS '20, December 2, 2020, Feldkirchen, Germany

(C) 2020 Copyright held by the owner/author(s).

ACM ISBN 978-1-4503-7621-1/20/06

https://doi.org/10.1145/3385958.3430483

\section{INTRODUCTION}

Automobiles are changing from driver-controlled and gasolinepowered vehicles to autonomous Electric Vehicles (EVs). Such vehicles will search for available Charge Points (CPs) and charge themselves using PnC protocols such as ISO 15118 [ISO/IEC 2013, 2014] or its successor ISO 15118-20 [ISO/IEC 2019]. While ISO 15118(-20) focuses on the communication between $\mathrm{EV}$ and $\mathrm{CP}$, the application guideline VDE-AR-E 2802-100-1 [VDE 2019] focuses on backend aspects such as the out-of-band (i.e., outside of the ISO 15118-20 specification) delivery of credentials. Together they enable a charging process with (nearly) no user intervention. If the vehicle is charged via a cable, only the cable must be plugged in. For inductive charging, the EV just parks over a coil.

The specified security mechanisms enable the EV to verify the authenticity of a CP operated by a Charge Point Operator (CPO), enable the EV to install contract credentials issued by the MO (i.e., the entity where the user has a charging service contract), and can use the charging services by authenticating itself using the contract credentials. PnC uses public-key authentication based on X.509 certificates and a dedicated Vehicle to Grid (V2G) Public Key Infrastructure (PKI). Before delivery, each EV gets a lifelong identity for PnC from its Original Equipment Manufacturer (OEM), a socalled OEM provisioning credential, which the driver registers at an MO when concluding a charging contract. The contract credentials, which are actually used for authenticating charging sessions, can either be installed during the first charging process (as defined in ISO 15118-20) or via an out-of-band mechanism (as defined in the application guideline) using the OEM provisioning credential. The respective private keys are created by the OEM and the MO in their backend systems making them vulnerable to attacks on corporate networks (cf. the Sony hack from 2014 ${ }^{1}$ ).

To address this issue, we already proposed HIP: HSM-based Identities for Plug-and-Charge [Fuchs et al. 2020a], a protocol extension for ISO 15118 where keys are generated and stored securely in a Hardware Security Module (HSM) (we propose to use a Trusted Platform Module (TPM) in our solution) within the EV and private keys never leave the HSM. HIP defines changes in the certificate installation/ update request message, a new custom X.509 v3 certificate extension for OEM provisioning and contract certificate, and requires some adaptions of the ISO 15118 protocol flow for

\footnotetext{
${ }^{1}$ https://www.wired.com/2014/12/sony-hack-what-we-know/
} 
credential provisioning. While HIP focuses on a backward compatible integration in the ISO 15118 process, it does not consider new certificate install/ update processes defined in ISO 15118-20, existing CA processes from vehicle manufactures with defined signing processes using CSRs [Cooper et al. 2008], or out-of-band certificate installations (as defined in the application guideline).

In this paper, we extend the original HIP to HIP-20 as an extension to ISO 15118-20 and application guideline VDE-AR-E 2802-1001. Similar to HIP, HIP-20 is backward compatible to ISO $15118-20$ and uses a TPM to securely store and use keys, with private keys never leaving the respective TPM preventing illegitimate copying to other EVs. In addition, HIP-20 enables easy integration into existing backend infrastructures and processes with support of existing CAs via CSRs and support of a CSR pool for timely distribution of CSRs. HIP-20 also does not require a contract certificate extension as in HIP, since ISO 15118-20 simplifies the certificate installation/update process. Our evaluation shows that HIP-20, just like HIP, significantly increases the security of $\mathrm{PnC}$ and can also be easily integrated into existing processes and infrastructures.

The remainder of this paper is organized as follows: We provide background on PnC, trusted computing, and the original HIP in Section 2. Related work is discussed in Section 3. Section 4 describes our system model and adversaries we consider. Security and functional requirements that HIP-20 should meet are defined in Section 5. In Section 6, we introduce the general concept of HIP-20 and evaluate HIP-20 in Section 7. We conclude the paper in Section 8.

\section{BACKGROUND}

In this section, we describe relevant background, i.e., security aspects of ISO 15118(-20), credential management via backend systems, relevant TPM concepts, and the idea of the original HIP.

\subsection{PnC Standards: ISO 15118(-20)}

The concept of Plug-and-Charge (PnC) was introduced by the standard ISO 15118 [ISO/IEC 2013], which defines a communication interface for controlling the energy transfer between an EV and a CP. $\mathrm{PnC}$ automates the installation of contract registration data, charging authorization, energy transfer, and billing of energy. ISO 15118-2 (part 2 of the 1st edition) [ISO/IEC 2014] specifies the protocol flow including message sequences and data formats. TLS, encryption, and digital signatures are used for security.

A new edition ISO 15118-20 [ISO/IEC 2019] will be published as final draft (FDIS) in 2021. This version is no longer fully backwards compatible. For reasons of backward compatibility, an updated ISO $15118-2$ is planned. New ISO $15118-20$ extends the scope to include additional use cases, e.g., bidirectional power transfer and wireless communication for conductive charging and wireless charging. The changed message formats for new service (re-)negotiations and metering confirmations are defined. Moreover, the processes change, e.g., the provisioning of the contract data is only possible using the OEM provisioning certificate. In addition, ISO 15118-20 defines TLS as mandatory for all use cases, updates the cipher suites and offers the option of billing for various contracts.

The main actors in data exchange for charging are still the Electric Vehicle Communication Controller (EVCC) and the Supply
Equipment Communication Controller (SECC). The secondary actors are still MO, Certificate Provisioning Service (CPS), OEM and CPO. The SECC must be able to pick up the contract credentials for the EV from the CPS, so that the EVCC can automatically install them. In order for the EVCC to charge and pay automatically, the CPO should enable the SECC to forward the EV's charging request to the relevant MO for authorization, if the SECC cannot check the contract credentials by itself. Another player is the OEM that ensures that the EVs it produces can install and renew contract credentials for future users. The OEM ensures that the EV is provided with a key pair $\left\{P C_{p u b}, P C_{p r}\right\}$ and a globally unique, lifelong identifier Provisioning Certificate Identifier (PCID) before it is delivered to the user. The OEM issues for the EV a provisioning certificate Cert $_{P C}$ that binds the public key $P C_{p u b}$ to the PCID. The EV user uses the PCID when concluding the charging contract, so that the EV can be assigned to a unique billing identity by the MO, the E-Mobility Account Identifier (EMAID), in the contract. The MO then issues contract data, including a key pair $\left\{C C_{p u b}, C C_{p r}\right\}$ and the contract certificate $\operatorname{Cert}_{C C}$ with the EMAID, encrypts the $C C_{p r}$ with the help of a session key created by an ephemeral-static ECDH key exchange. The MO's session key is formed from an ephemeral part $\left\{\right.$ DHKey $\left.y_{p u b}, D H K e y_{p r}\right\}$ and a static part $P C_{p u b}$ of the EV's OEM provisioning certificate. The MO packs $D H K e y_{p u b}$ and the MO's SubCA certificate chain together with the mentioned contract data and forwards the newly created contract data package for validation and provisioning to the CPS. The CPS acts as a trustworthy provider of contract data packages from several MOs for different EVs from one or more OEMs. The CPS validates contract data packages, builds installable responses from them, signs the responses and saves them securely under the PCID of the recipient.

If the EVCC is connected to the SECC and has no valid contract credentials, the EVCC can automatically request the contract data packages from the CPS via the ISO 15118 connection to the SECC. The EVCC uses its OEM provisioning certificate and the corresponding private key $P C_{p r}$ to authenticate its request to the CPS and to retrieve its contract data packages. The EVCC unpacks its contract data packages, decrypts the private keys $C C_{p r}$ and installs the credentials. The EVCC uses the installed contract credentials to activate the charging service and for billing via the respective contract. The ultimate authorization of the charging service is generally carried out by the MO itself outside of ISO 15118, since only the MO can check the status of the EV's contract.

\subsection{Backend Aspects: VDE-AR-E 2802-100-1}

The application guideline VDE-AR-E 2802-100-1 [VDE 2019] of the German standardization organization DKE aims to address open specification gaps of ISO 15118-2 for the exchange of digital certificates and key materials between backend actors. Also, technical measures for installing contract credentials in the vehicle including alternative processes, e.g., OEM links are considered. In addition to the standard ISO 15118 communication, the guideline allows intermediate storage of root $\mathrm{CA}$ certificates and $\mathrm{PnC}$ credentials in a central data store, so that the processes of the actors in the charging ecosystem can run independently of one another.

This solution (cf. Fig. 5) uses such a Global Certificate Store (GCS) to allow MOs to generate contract credentials and make them 
available in advance, before they are requested by the vehicle. This helps meet strict timing limits of ISO 15118 for their delivery. The GCS consists of OEM Provisioning Certificate Pool and Contract Certificate Pool. The OEM Provisioning Certificate Pool enables the data exchange between OEMs and MOs. The OEM uploads vehicle data including OEM provisioning certificates into this Pool, where they are stored under respective PCIDs. When the MO receives the PCID from the user, it can directly extract all information necessary for generating the contract credentials for this EV from the Pool. Having generated the contract credential, the MO transfers it within a contract certificate package to the CPS for signing. After being signed, the package is sent to the Contract Certificate Pool, where it is stored under the EMAID, PCID, and the XML schema definition. The Contract Certificate Pool provides data exchange between the CPO/SECC and CPS as well as OEM and CPS. When the SECC receives a certificate installation request from the EVCC, it can download the respective contract credential from the Pool and deliver it to the vehicle without delay. Alternatively, the OEM can collect contract credentials for own vehicles from the Pool and deliver them via an OEM proprietary link to the EV. This way, the generation and delivery of contract credentials can take place out-of-band, i.e., outside of the ISO 15118 protocol flow.

\subsection{Trusted Platform Modules (TPMs)}

The TPM 2.0 Library Specification [Trusted Computing Group 2014] defines the functionalities for building TPMs for different platforms. The TPM is mostly realized as a dedicated hardware chip and provides a specially shielded area for secure storage and usage of keys. Thus, it is a good choice for security-critical applications such as payment transactions. Most TPM chips have a security certification, e.g., Common Criteria (CC) EAL4+, and some are also qualified according to the automotive AEC-Q100 standard. Besides the secure storage and usage feature, we use the credential protection and enhanced authorization functionality of the TPM in our solution. Below we list the relevant TPM 2.0 commands and policy elements:

- TPM2_Create This command is used to create various objects for the TPM. This includes cryptographic keys usable for authenticating to external entities. During creation, an (enhanced authorization) policy can be defined to restrict usage of the created object.

- TPM2_Sign This command calculates a signature using a private key created via TPM2_Create that can be used to authenticate a device or to assert data integrity and origin.

- TPM2_PolicyAuthorize This command allows the activation of policies after the definition of an object. In order to achieve this, a public key is registered with a policy. This policy element then acts as a placeholder for any other policy branch that is signed with the corresponding private key.

- TPM2_PolicyPCR This policy statement ensures that the Platform Configuration Registers (PCRs) have a certain value. The PCRs are used to represent the integrity state of a platform by recording its booted software and configurations.

- TPM2_ActivateCredential This command is part of the TPM credential deployment capabilities. A credential provider can encrypt a certificate with a key known to reside on the TPM and denote a certain additional key inside the encrypted data. The TPM decrypts the data and checks that the denoted key is known and only then hands the credential data back to the user. Thus, the credential provider has assurance that credential usage is only possible if the denoted key is known to the same TPM with the reported properties.

\subsection{HIP ISO 15118 Extension}

In [Fuchs et al. 2020a], we proposed HIP, a protocol extension for ISO 15118 where keys are generated and stored securely in a TPM within the EV. Private keys never leave the TPM. HIP defines minor changes in the certificate installation/update request message, a new custom X.509 v3 certificate extension, and requires some adaptions of the ISO 15118 protocol flow for credential provisioning.

Before delivery, the key pair $\left\{P C_{p u b}, P C_{p r}\right\}$ of the OEM provisioning credential is generated by the TPM and the OEM creates the corresponding OEM provisioning certificate Cert $P C$. When an EV connects to a $\mathrm{CP}$ and wants to install (or update) contract credentials, the TPM generates the key pair $\left\{C C_{p u b}, C C_{p r}\right\}$. The EVCC then sends a request via $\mathrm{CP}$ to $\mathrm{MO}$ for issuing the corresponding contract certificate Cert $_{C C}$ for the public key $C C_{p u b}$. After receiving Cert $_{C C}$, EVCC uses $C C_{p r}$ for normal ISO 15118 operations.

HIP uses several TPM features. Secure key storage and usage ensures that private keys cannot be misused by an adversary. The secure credential activation feature ensures that $C C_{p r}$ is actively usable only on exactly the TPM where it has been generated. Enhanced authorization enables access control to keys, e.g., access is only possible if the EVCC has booted in a trustworthy state. HIP must be supported only by OEM, EVCC, and MO. Intermediate actors such as a CP simply forward the (extended) messages.

\section{RELATED WORK}

Many research activities deal with the security of modern vehicles. Different attack vectors have been analyzed in [Checkoway et al. 2011], attacks via the wireless tire pressure monitoring systems in [Rouf et al. 2010], attacks via a diagnostics port in [den Herrewegen and Garcia 2018; Miller and Valasek 2014a], or remote attack vectors in [Miller and Valasek 2014b]. Remotely controlling a vehicle was discussed in [Koscher et al. 2010] and demonstrated in practice in [Greenberg et al. 2015]. These attacks show that access to sensitive data is possible if no special hardware is used.

Automotive HSMs such as the SHE module [HIS 2009] or the HSMs defined in the European project EVITA are often implemented as System on Chip (SoC). Based on the results of the EVITA project, several suppliers developed automotive micro controllers with integrated HSMs for securing the in-vehicle communication, e.g., for Secure Onboard Communication (SecOC) on the CAN bus [AUTOSAR 2017] or TLS on automotive Ethernet [Zelle et al. 2017]. These HSMs provide some basic protection against illegitimate readout of data but usually do not provide some specially shielded area for secure storage with protection against more sophisticated sidechannel attacks. This is also the case with the HSMs for Vehicle2X (V2X) communication, as specified by the Car 2 Car Communication Consortium [CAR 2 CAR Communication Consortium 2018].

Using a TPM in vehicles has been proposed in related work. In [Fuchs et al. 2016], a TPM is used in a head unit to realize protocols for secure update, remote attestation, and sensitive data 
protection. In [Xu et al. 2018], the authors propose a TPM-based remote attestation for identity and integrity verification in V2X communication. Using a TPM in EVs was also already discussed. In [Zelle et al. 2018; Zhao et al. 2015], solutions for preventing the generation of movement profiles based on the charging data using a TPM and Direct Anonymous Attestation (DAA) protocols are proposed. Privacy-aware architectures for vehicle-to-grid networks are presented in [Ghosh et al. 2013] and [Saxena et al. 2017]. As part of these solutions, a TPM was used in EV batteries for encrypted communication of charging status, accumulation of information in sealed storage and remote attestation. A TPM-based security solution which ensures that only a vehicle with a manufacturer approved charging system can connect to the power grid is proposed in [Fuchs et al. 2020b]. Secure value-added-services for EVs using TPM are proposed in [Buschlinger et al. 2019]. In [Fuchs et al. 2020c], the security architecture TrustEV for secure provisioning, storage, and usage of ISO 15118 PnC credentials based on the TPM 2.0 is introduced. TrustEV does not support local key generation directly in the TPM, which is addressed in HIP [Fuchs et al. 2020a].

\section{SYSTEM AND THREAT MODEL}

Our system model adopts the actors from ISO 15118-20 and application guideline as described in Sections 2.1 and 2.2. We consider EVCC and SECC as main actors. In addition, a TPM provides security services for the EVCC. Secondary actors are the vehicle OEM, the GCS with the certificate pools, the MO's CA issuing contract credentials and the MO's ISO 15118-20 handler encrypting them for delivery, and the CPS enabling their delivery to the EVCC.

Our threat model considers an adversary who aims to extract or copy the $\mathrm{PnC}$ credentials of an EV, i.e., OEM provisioning and contract certificate(s) along with their private keys, and to use them for profit (e.g., to impersonate the legitimate user or to forge bills). The attack surface includes the EVCC and the SECC, the backend systems of GCS, CPS, CAs, and the MO's ISO handler as well as their network connections. With ISO 15118-20, OCPP 2.0, and the application guideline demanding the use of TLS, we assume the communication to be secure under the Dolev-Yao adversary [Dolev and Yao 1983] and consider attacks via the network channel and any attacks on cryptography out of scope.

We assume the following two types of adversaries. The first type is a local adversary who has full physical access to the EVCC and can extract any data except for HSM-protected data. The adversary can also try to trick the HSM to perform operations such as signing certificate requests. The second type of adversary has compromised a backend system responsible for issuing or delivery of PnC credentials.

\section{REQUIREMENTS FOR HIP-20}

To prevent the attacks on the $\mathrm{PnC}$ authentication and to mitigate the risks for customer accounts in the backend systems, we propose to create and store OEM provisioning and contract credentials in the HSM of the customer's EV. This way, the credentials are out of reach for backend hackers. On the downside, the credential generation in the vehicle now has to meet security standards expected by the MO Moreover, the MO may demand the EV to prove that the contract credentials were generated and stored securely. To achieve this goal, several security requirements should be met as detailed below.

$R S_{1}$ Secure key generation: Cryptographic keys for ISO 15118-20 shall be generated within a protected and secure environment within the EVCC they are destined for. Similar, all required random numbers, e.g., for key generation, shall also be generated within this environment. This environment shall be physically separated from the other components.

$\mathrm{RS}_{2}$ Secure key storage: Private ISO 15118-20 keys shall also be stored in the protected and secure environment to prevent their leakage.

$\mathrm{RS}_{3}$ Secure cryptographic operations: To protect cryptographic keys from leakage during usage, the protected and secure environment shall also provide a secure execution environment for cryptographic operations.

$R S_{4}$ Key usage authorization: To prevent arbitrary access to the private keys by an unauthorized service on the EVCC's processor or a tampered firmware, access to these keys shall be limited to authenticated and trusted systems.

$R S_{5}$ Cryptographic agility: Insecure cryptographic primitives and algorithms shall be updateable and exchangeable.

$R S_{6}$ Trustworthy credential enrollment: An EV shall be able to use the contract certificate issued for it by an MO only if the respective private key has been generated in a secure environment of this vehicle and with the expected parameters.

Though ISO 15118-20 is yet under development, its current final draft status does not permit substantial technical changes. Moreover, this new edition will use the same backend infrastructure now being built upon ISO 15118-2 [ISO/IEC 2014] and DKE application guideline [VDE 2019]. Therefore, the acceptance of the proposed solution strongly depends on the fulfillment of the following functional requirements:

$R F_{1}$ Minimal overhead: Additional communication and computational overhead shall be kept possibly low compared to the ISO 15118-20 protocol flow. The overhead should at least not result in data size limits or time limits of being exceeded.

$\mathrm{RF}_{2}$ Conformance to ISO 15118-20 [ISO/IEC 2019]: The ISO 1511820 message sequences shall be preserved. Changes to the ISO 15118-20 message formats shall be minimal; newly introduced elements shall be optional.

$R_{3}$ Backwards compatibility: The solution shall not impede the operation of any actor compliant with ISO 15118-20, even if this actor does not support the proposed security extension.

$R_{4}$ Conformance to DKE application guideline [VDE 2019]: The solution shall permit out-of-band certificate installation using intermediate storage (certificate pools) between the various IT instances of the actors in the ecosystem and the alternative delivery of contract information via the OEM's backend.

$R F_{5}$ Conformance to standard $C A$ procedures: EVCC, CPS, $\mathrm{CP} / \mathrm{CPO}$, OEM backend and the issuer $(\mathrm{MO})$ of contract certificates shall support at least the standard procedure for applying for, checking and issuing PKIX certificates according to RFC 5280 [Cooper et al. 2008] with CSRs. In detail, this means that these parties have to support both the initial issue and the renewal of contract certificates in this way. 


\section{CONCEPT OF HIP-20}

In this section, we describe the concept of our ISO 15118-20 protocol extension HIP-20. First, we describe the differences to the original HIP and the general idea of HIP-20. Then we describe the new components of HIP-20 and the integration of HIP-20 in the protocol flows of ISO 15118-20 and the DKE application guideline.

\subsection{Improvements Introduced with HIP-20}

HIP-20 has the following differences to the original HIP [Fuchs et al. 2020a]. The details are provided in the following sections.

Use of CSR in CertInstallReq. Instead of sending the plain public key $C C_{p u b}$ as part of the CertInstallReq, a standard compliant CSR is sent. This improves compatibility with existing CAs.

Publishing of CSRs in a CSR pool. The EVCC can send CSRs in advance to the OEM, e.g., via a telematics link. The OEM collects these CSRs in a special data store (pool) in the backend from where MOs can retrieve them in a read-and-delete operation. This gives the $\mathrm{MO}$ more time to generate contract credential certificates $\mathrm{Cert}_{C C}$ for TPM-generated credentials. This is similar to the current pool approach for provisioning credential certificates $\operatorname{Cert}_{P C}$.

Optional ignoring of CSRs. CPS/MO is allowed to ignore the CSR sent by the EVCC as part of the CertInstallReq and to provide a different certificate Cert $_{C C}$ as part of the CertInstallRes instead. This certificate would be derived from a CSR of the CSR pool.

Encryption of the certificate signature only. Only the signature of the Cert $_{C C}$ is encrypted instead of the whole certificate. This allows the EVCC to detect whether an ISO-compliant Cert $_{C C}$ was issued, based on its CSR or whether the CSR was ignored and a Cert $_{C C}$ based on a pool CSR was issued.

No TPM extension in contract credential. The contract credential certificate $\mathrm{Cert}_{\mathrm{CC}}$ does not contain an extension anymore since ISO 15118-20 always uses the $\operatorname{Cert}_{P C}$ for Cert $_{C C}$ installation/update. Only $\operatorname{Cert}_{P C}$ needs to contain a TPM extension.

\subsection{General Idea of HIP-20}

The general idea of HIP-20 is to create keys securely in the TPM so that the private key never leaves the TPM. Before vehicle's delivery, the key pair $\left\{P C_{p u b}, P C_{p r}\right\}$ is generated by the TPM and the OEM creates the corresponding OEM provisioning certificate Cert $_{P C}$. Similarly, the TPM generates the key pair $\left\{C C_{p u b}, C C_{p r}\right\}$ and the $\mathrm{EVCC}$ requests the $\mathrm{MO}$ to issue the corresponding contract certificate Cert $_{C C}$ for the public key $C C_{p u b}$.

Fig. 1 illustrates the general idea of HIP-20 for the contract credential provisioning. Whereas in regular ISO 15118-20 the MO generates keys and corresponding certificate and sends them to the EVCC (Fig. 1a), HIP-20 allows the EVCC to generate contract keys locally inside its TPM and request corresponding certificates from the MO over ISO 15118 (Fig. 1b). Note that a PKCS \#10 CSR [Nystrom and Kaliski 2000] is used to request the Cert $_{C C}$ as this is a common requirement from CAs. For instance, the certificate policy for the V2G PKI of Hubject (the most established CA for EV charging [Berman 2020]) only allows two methods for credential provisioning [Hubject $\mathrm{GmbH} 2020$ ]: (i) with key generation by the CA and (ii) via a PKCS \#10 CSR.

We use the TPM to ensure secure key storage and usage. The secure credential activation feature ensures that $C C_{p r}$ is actively

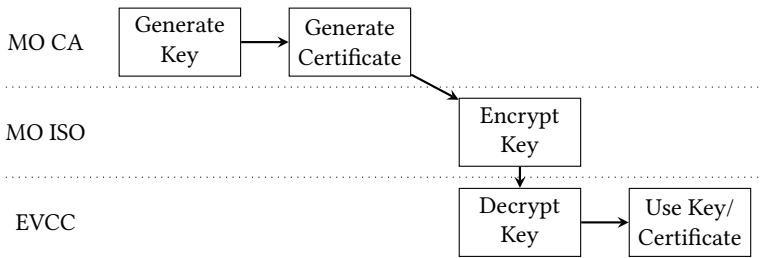

(a) ISO 15118-20

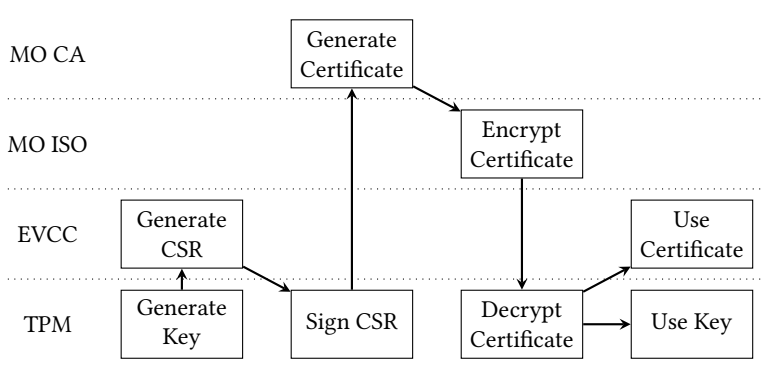

(b) Protocol Extension HIP-20

Figure 1: Contract Credential Provisioning

Table 1: Public Area Attributes of the TPM 2.0 Keys

\begin{tabular}{|l|l|l|l|}
\hline Attribute & \multicolumn{1}{|c|}{ SRK } & \multicolumn{1}{|c|}{ PC } & \multicolumn{1}{c|}{ CC } \\
\hline type: & \multicolumn{3}{|c|}{ TPM2_ALG_ECC } \\
\hline nameAlg: & \multicolumn{3}{|c|}{ TPM2_ALG_SHA256 } \\
\hline object- & $\begin{array}{l}\text { fixedTPM, fixedParent, } \\
\text { Attributes: } \\
\text { restricted, decrypt, } \\
\text { sensitiveDataOrigin, } \\
\text { userWithAuth, noDA }\end{array}$ & $\begin{array}{l}\text { fixedTPM, } \\
\text { fixedParent, } \\
\text { sign, decrypt, } \\
\text { sensitiveDataOrigin }\end{array}$ & $\begin{array}{l}\text { fixedTPM, } \\
\text { fixedParent, } \\
\text { sign, } \\
\text { sensitiveDataOrigin }\end{array}$ \\
\hline authPolicy: & \multicolumn{3}{|c|}{ n/a TPM2_ECC_NIST_P256 } \\
\hline curveID: & \multicolumn{3}{|c|}{ TPMAuthorize } \\
\hline
\end{tabular}

usable only on exactly the same TPM where it has been generated. The enhanced authorization feature of the TPM (cf. Section 3) is used for controlling access to keys by defining policies. For example, access to keys may only be possible if the EVCC has booted in a trustworthy state and nobody has tampered with the firmware.

To enable the use of HIP-20 with a TPM, only OEM, EVCC, and the ISO 15118-20 handler of the MO need to implement the additional security features. HIP-20 can be transparently used by intermediate actors such as a CP and MO CAs. HIP-20 requires changes in the ISO 15118-20 certificate installation request message, a new X.509 v3 certificate extension, and some adaptions of the ISO 15118-20 protocol flow for provisioning contract credentials. In the following sections, we describe HIP-20 in detail.

\subsection{Definition of Components}

6.3.1 TPM 2.0 Keys. With HIP-20, all keys used for PnC are generated inside the EVCC's TPM. They are protected by a TPM policy to be defined by the OEM. Table 1 shows the TPM keys used by HIP-20: the OEM provisioning key pair $\left\{P C_{p u b}, P C_{p r}\right\}$, the contract credential key pair $\left\{C C_{p u b}, C C_{p r}\right\}$, and a TPM storage root key $\left\{S R K_{p u b}, S R K_{p r}\right\}$ which is used for storing the other keys. All keys are ECC key pairs and use the secp256r1 curve. 


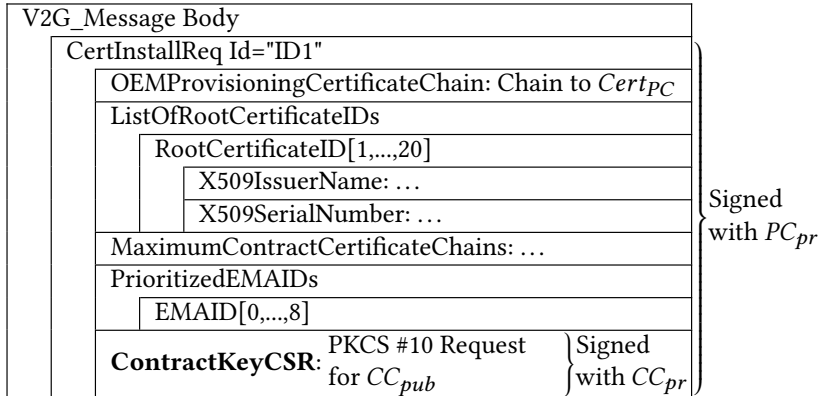

Figure 2: Extension in ISO 15118-20 CertInstallReq message

The SRK's profile follows the TCG's recommendation for a default storage primary key template [Trusted Computing Group 2017]. The $P C$ and $C C$ keys carry the TPM objectAttributes fixedTPM and fixedParent to forbid the private key export (cf. [Trusted Computing Group 2016], Section 25) and sensitiveDataOrigin to denote that they were generated by the TPM and not imported. The attributes sign and decrypt denote the usage type for these keys. We use TPM2_PolicyAuthorize as default policy that allows for policies to change by associating the policy to a signing authority and allowing the policy contents to change. The OEM can decide to set another policy, e.g., the TPM2_PolicyPCR can be used to create a policy enabling access to the keys only if the EVCC's firmware is in a trustworthy state which has been verified in a measured boot (cf. [Trusted Computing Group 2016], Section 19.7 Enhanced Authorization). In contrast to the $S R K$, the restricted attribute is not set since $P C$ and $C C$ shall serve as general-purpose keys and not storage keys.

6.3.2 ISO 15118-20 certificate installation request message. The only required change to ISO 15118's message definitions is the inclusion of a PKCS \#10 CSR for the public contract key $C C_{p u b}$ within the EVCC's certificate installation CertInstallReq request message. The CSR is signed with $C C_{p r}$ (cf. [Nystrom and Kaliski 2000]) providing a proof-of-possession of this private key. Since the EVCC does not know the correct EMAID yet, the subject name field is omitted in the CSR and added in Cert $_{C C}$ by the MO CA.

We extend the definition of the CertInstallReq message with the optional field ContractKeyCSR, encoded as a Base64 string. Since this field is part of the CertInstallReq body, it is also included in the XML signature with $P C_{p r}$ for these messages and does not require any special treatment. Fig. 2 shows the changed CertInstallReq.

ISO 15118 includes a protocol negotiation step based on a major and minor version number. The SECC must allow and forward a connection from an EVCC even with a higher minor version, as long as the major version matches. In such a case, the SECC shall ignore but forward additional or unknown data elements sent by the EVCC (cf. [ISO/IEC 2019], Section 8.2.1). Since the SECC does not need to process any information from certificate requests, it simply forwards them to the MO. The additional field can be represented in a minor version number. Thus, changes in any intermediate nodes such as the SECC are not required.

\begin{tabular}{|c|c|c|}
\hline \multicolumn{3}{|c|}{ OEM Provisioning Certificate $\left(\mathrm{Cert}_{P C}\right)$} \\
\hline \multicolumn{2}{|r|}{ Version: } & X.509v3 (0x2) \\
\hline \multicolumn{2}{|r|}{ Serial Number: } & $12345(0 \times 3039)$ \\
\hline \multicolumn{2}{|c|}{ Signature Algorithm: } & ecdsa-with-SHA256 \\
\hline \multicolumn{2}{|r|}{ Issuer: } & $\mathrm{CN}=\mathrm{OEMSubCA} 2, \mathrm{O}=\mathrm{ISO}$ \\
\hline \multirow{2}{*}{ Validity } & Not Before: & May 7 08:40:32 2020 GMT \\
\hline & Not After: & May 6 08:40:32 2050 GMT \\
\hline \multicolumn{2}{|r|}{ Subject: } & $\mathrm{CN}=8 \mathrm{AAA} 2 \mathrm{~B} 3 \mathrm{C} 4 \mathrm{D} 5 \mathrm{E} 6 \mathrm{~F} 7 \mathrm{G} 92, \mathrm{O}=\mathrm{ISO}$ \\
\hline \multirow{3}{*}{$\begin{array}{c}\text { Subject } \\
\text { Public } \\
\text { Key Info }\end{array}$} & Public Key: & OCTET STRING $\left(P C_{p u b}\right)$ \\
\hline & Algorithm: & id-ecPublicKey \\
\hline & Parameters: & namedCurve secp256r1 \\
\hline \multirow{10}{*}{$\begin{array}{c}\text { X509v3 } \\
\text { Exten- } \\
\text { sions }\end{array}$} & Basic Constraints: $^{\mathrm{C}}$ & CA:FALSE \\
\hline & Key Usage: ${ }^{\mathrm{c}}$ & Digital Signature, Key Agreement \\
\hline & Subject Key Identifier: $^{\mathrm{c}}$ & 64 bit ID of $P C_{p u b}$ \\
\hline & Authority Key Identifier: ${ }^{\mathrm{C}}$ & 64 bit ID of issuer's public key \\
\hline & CRLDistributionPoints: $^{\text {nc }}$ & URI:http://example.com/example.crl \\
\hline & $\begin{array}{l}\text { Authority Information } \\
\text { Access (OCSP): }{ }^{\text {nc }}\end{array}$ & URI:http://ocsp.example.com/ \\
\hline & \multirow{4}{*}{$\begin{array}{l}\text { Subject Information } \\
\text { Access: }\end{array}$} & OID:1.0.20.4 \\
\hline & & $\begin{array}{l}\text { TPM 2.0 EC Storage Root Key }\left(S R K_{p u b}\right) \\
512 \text { bit OCTET STRING in Base64 }\end{array}$ \\
\hline & & OID:1.0.20.5 \\
\hline & & $\begin{array}{l}\text { TPM 2.0 SHA256 Policy Digest }(\mathrm{Pol}) \\
256 \text { bit OCTET STRING in Base64 }\end{array}$ \\
\hline Signature & \multicolumn{2}{|c|}{$\begin{array}{ll}\text { Algorithm: } & \text { ecdsa-with-SHA256 } \\
\text { Value: } & \text { OCTET STRING } \\
\end{array}$} \\
\hline
\end{tabular}

Figure 3: Provisioning Certificate with TPM Extension

6.3.3 Certificate TPM Extension. In order to encrypt the signature of generated contract certificates such that only the TPM can decrypt it, the MO requires some additional information; the $S R K_{p u b}$ and the TPM policy Pol of the $C C$. The $S R K_{p u b}$ is used as decryption target and $\mathrm{Pol}$ is used as part of the credential datatype encryption. The authenticity and integrity of this data is important to ensure compliance with the defined policy of the OEM. They are sent to the MO as part of the OEM provisioning certificate $\operatorname{Cert}_{P C}$. This approach ensures that keys for the contract certificates are created in the EVCC's TPM with defined attributes and bound to a policy defined by the OEM.

These two informational elements are encoded in a Subject Information Access X.509 certificate extension as Base64 strings using the rfc822Name type as defined in [Cooper et al. 2008] (cf. Fig. 3 ). The extension is included in the OEM provisioning certificate Cert $_{P C}$ and marked as non-critical (nc) in order to preserve backwards compatibility with MOs not supporting this extension. The first element (identified by OID 1.0.20.4) carries the public portion of the EVCC's TPM SRK and the second one (identified by OID 1.0.20.5) includes the TPM policy Pol, represented as a SHA256 digest value.

The remainder of the certificate is not altered and follows the certificate profile specified in ISO 15118-20 (cf. [ISO/IEC 2019], Annex B). Note that ISO 15118-20 already uses a Subject Information Access extension in contract certificates to carry custom information about the user's contract with the MO (also using the rfc822Name type). Hence, using this extension in provisioning certificates only leads to minimal new requirements for V2G CAs.

\subsection{Integration of HIP-20 in the ISO 15118-20 Protocol Flow}

6.4.1 Preparation of contract-based charging. Before delivery of the vehicle, the EVCC's TPM creates the storage root key SRK 
used for securely storing all other keys and the OEM provisioning key pair $P C$ is stored "under" the $S R K$ according to the templates in Section 6.3.1. The public keys $S R K_{p u b}$ and $P C_{p u b}$ are read out by the OEM in a secure way. Thus, the OEM shall be certain that these keys actually belong to the device being manufactured. The TPM policy for the $P C_{p u b}$ can be set to anything suitable for the OEM's security policies for usage of these keys. It could be bound to certain firmware integrity states using TPM2_PolicyPCR, being extendable by using TPM2_PolicyAuthorize (cf. Section 6.3.1 and [Trusted Computing Group 2016], Section 19.7.11), or a combination of several policies.

The OEM then generates the OEM provisioning key certificate Cert $_{P C}$ including the additional informational elements $S R K_{p u b}$ and $\mathrm{Pol}$ (cf. Section 6.3.3). The MO uses these elements in the encryption of a contract certificate's signature as described below.

6.4.2 Contract credential installation. The process for installing contract credentials with HIP-20 using the modified CertInstallReq message is illustrated in Fig. 4a.

First, the TLS channel between EVCC and SECC is established and the SECC authenticates itself (not shown). Next, the EVCC calls the TPM to generate a new key pair $\left\{C C_{p u b}, C C_{p r}\right\}$ and creates the corresponding CSR with $C C_{p u b}$ (also not shown). Note that the CSR is signed with $C C_{p r}$ (cf. [Nystrom and Kaliski 2000]), which requires the contract key's TPM policy to be met. Next, the EVCC generates a CertInstallReq message containing Cert ${ }_{P C}$ (including $S R K_{p u b}$ and $P o l$ ) and the CSR in the new field (cf. Section 6.3). It calls the TPM with the TPM2_Sign() function to load $P C$ and use $P C_{p r}$ for signing the message. The TPM first checks whether the defined policy is met (PC_PolicyCheck()) and then returns the signed CertInstallReq which is forwarded via the SECC to the MO.

On receiving the CertInstallReq message, the MO verifies the signature using $P C_{p u b}$. If the MO does not support HIP-20, it ignores the additional field in the request as well as the X.509 extension field in the OEM provisioning certificate, as specified by ISO 15118-20. Instead, it follows the regular approach of generating a new contract key pair with a corresponding contract certificate and sending it to the EVCC. If the MO supports HIP-20, the signature of the CSR is verified with the included $C C_{p u b}$ and the certificate generation for $C C_{p u b}$ is started, resulting in the contract certificate Cert $_{C C}$. In addition, a symmetric AES key $S K$ is generated and used to encrypt the signature of $\operatorname{Cert}_{C C}$ (shown as $E n c_{S K}\left(\operatorname{Cert}_{C C}\right.$ ) in Fig. 4a). SK itself is then encrypted with $S R K_{p u b}$, i.e., with another symmetric key generated by ephemeral-static ECDH using the static $S R K_{p u b}$ and a newly generated ephemeral $D H K e y_{p r} .^{2}$ More precisely, the $S K$ is embedded in a TPM specific data structure together with a set of information for usage by the TPM. For this, the MO calculates $C C_{\text {name }}$, a hash digest calculated over the objectAttributes of $C C_{p u b}$ (cf. Table 1), the defined policy of the OEM Pol (cf. Fig. 3), and $C C_{p u b} . C C_{\text {name }}$ is used in the encryption of $S K$ to ensure that $C C_{p u b}$ has been generated with the expected attributes and policy in the same TPM as the used $S R K_{p u b}$.

\footnotetext{
${ }^{2}$ More precisely, an ECDH key pair $\left\{\right.$ DHKeypub,$\left.D H K e y_{p r}\right\}$ is generated and secret $Z$ is calculated from $D H K e y_{p r}$ and $S R K_{p u b} . Z$ is then used with a KDF to generate an encryption and an integrity key. $S K$ is encrypted with the encryption key and an HMAC is computed with the integrity key over the encrypted data.
}

The CertInstallRes message containing the $\operatorname{Enc}_{S K}\left(\operatorname{Cert}_{C C}\right)$, the encrypted $S K$, and the public $D H K e y_{p u b}$ is signed with the private key $M O_{p r}$ of the MO (if MO takes the role of CPS; otherwise, with $C P S_{p r}$ of the CPS) and sent to the EVCC via the SECC.

The EVCC calls the TPM with the TPM2_ActivateCredential to load $C C$ and $S R K$. The TPM uses $S R K_{p r}$ to decrypt $S K^{3}$ and then returns $S K$ to the EVCC that can in turn decrypt the signature of Cert $_{C C}$ with AES and start using the certificate. Note that the TPM will only perform the decryption operation, if both, the $S R K_{p r}$ and the $C C_{p r}$ are loaded at the same point in time; thus acknowledging a binding between those two entities.

6.4.3 Contract credential usage. After decrypting the signature of Cert $_{C C}$, the EVCC can use the private contract key $C C_{p r}$ stored in the TPM for all normal ISO 15118 use cases. If the decryption fails, Cert $_{C C}$ is basically voided. For instance, for $\mathrm{PnC}$ authorization the EVCC receives a nonce from the SECC and responds with a signature (created with $C C_{p r}$ ) over the nonce and Cert $_{C C}$ (and certificate chain of MO Sub-CA certificates). Only if the signature can be verified with $C C_{p u b}$ from Cert $_{C C}$ and the $\operatorname{Cert}_{C C}$ chain can be traced up to a trusted root $\mathrm{CA}$, which requires the signature of Cert $_{C C}$ to be verifiable (i.e., decrypted), is the vehicle cleared to charge. The only difference of HIP-20 in the PnC authorization process is that creating a signature with $C C_{p r}$ requires interaction with the TPM as well as the correct assertion of the key's policy. The same change is introduced in all other ISO 15118-20 processes that use the private contract key (i.e., signing of metering receipts).

The process of using $C C_{p r}$ depends on the specific policy, which the OEM provided for the EVCC. For the example of TPM2_PolicyAuthorize used to authorize a TPM2_PolicyPCR, the EVCC's local software state is recorded during a measured boot and stored in one of the TPM's Platform Configuration Registers (PCRs). Then, the EVCC loads the signed TPM2_PolicyPCR digest (PolPCR; provided by the OEM alongside the current firmware) and verifies the signature using the OEM's public key. The result is a ticket, proving the successful signature verification by the TPM.

For performance improvements, the EVCC may acquire and store policy signature verification tickets from its TPM before they are needed in a policy. This omits costly asymmetric operations during credential usage. The EVCC starts a policy session and executes the TPM2_PolicyPCR command. The intermediate policy digest must be equal to $P_{P C R}$, signed by the OEM. Afterwards, the EVCC executes the TPM2_PolicyAuthorize command, providing the verified Pol $_{P C R}$ and the ticket of verification. Only if the intermediate policy is equal to $P o l_{P C R}$, i.e., the local software state is as expected, is the assertion successful and the EVCC's contract key can be used.

\subsection{Integration of HIP-20 in the VDE-AR-E 2802-100-1 Infrastructure}

HIP-20 can also be implemented in combination with the DKE application guideline [VDE 2019] without further changes to ISO 1511820 being necessary. Only the guideline and the auxiliary processes need to be adjusted as shown in Fig. $4 \mathrm{~b}$ and Fig. 5 .

6.5.1 Preparation of contract-based charging. The DKE application guideline allows the individual actors to run their processes

\footnotetext{
${ }^{3}$ As mentioned above, $S R K_{p r}$ is not used directly but symmetric session keys.
} 


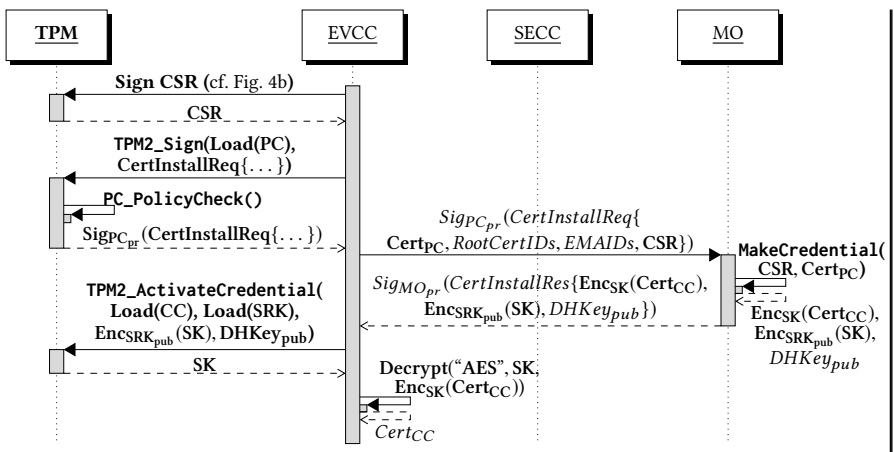

(a) Provisioning over ISO 15118-20

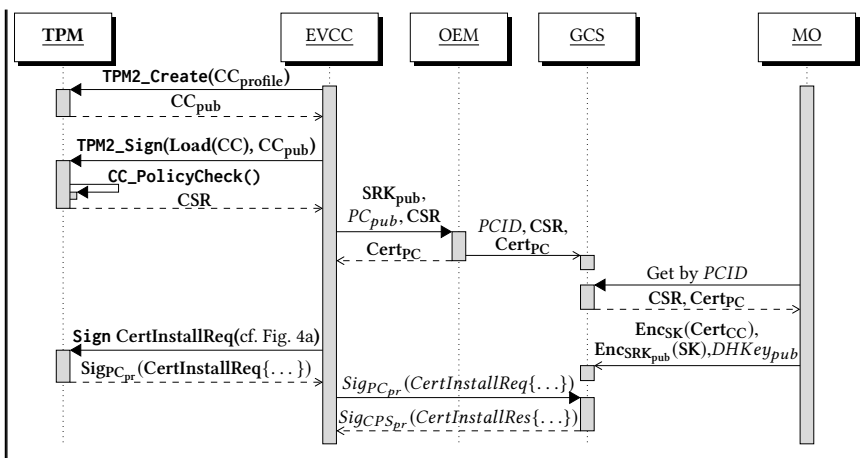

(b) Provisioning over VDE-AR-E 2802-100-1

Figure 4: HIP-20 Provisioning of Contract Credentials (Extensions to Standards in Bold)

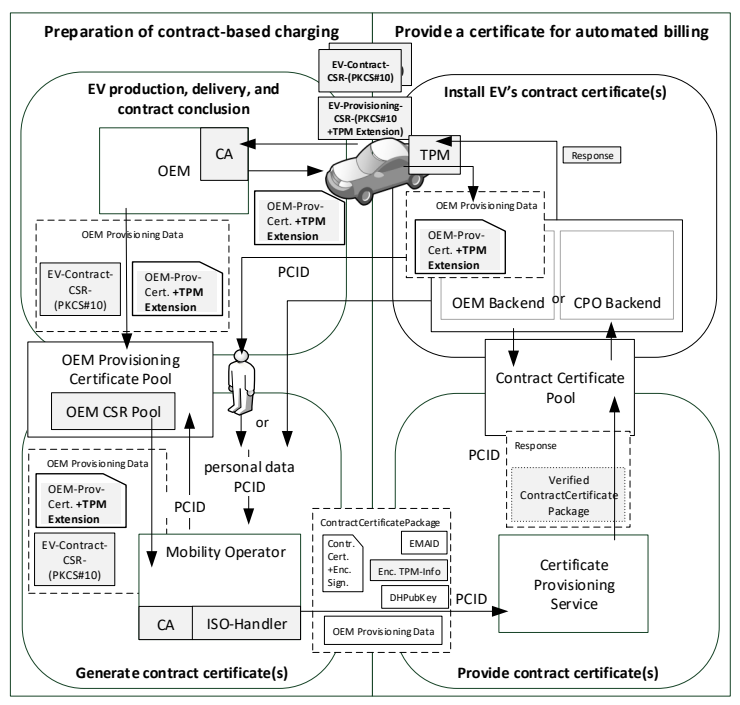

Figure 5: HIP-20 with VDE-AR-E 2802-100-1

concurrently and independently of one another by introducing intermediate data storages (pools) (cf. Section 2.2). Consequently, the OEM must now ensure that the additional HIP-20 data from the TPM end up in a pool before processing. Therefore, the so called OEM CSR Pool is added as part of the OEM Provisioning Certificate Pool to store these data. Hence, the preparation of EVCCs (cf. Section 6.4.1) is changed as follows: The EVCC's TPM regularly generates contract key pairs $\left\{C C_{p u b}, C C_{p r}\right\}$ and signs CSRs for the corresponding public keys $\left(C C_{p u b}^{1}, \ldots, C C_{p u b}^{n}\right)$. The data necessary for issuing the contract certificate, such as the CSRs and other vehicle data ( Cert $_{P C}$ including $S R K_{p u b}$ and Pol, PCID, etc.) are read out by the OEM and uploaded into the OEM CSR Pool.

6.5.2 Provide a contract certificate. After the conclusion of a contract between $\mathrm{EV}$ user and $\mathrm{MO}$, the $\mathrm{MO}$ reads the $\operatorname{Cert}_{P C}$ and reads and deletes a CSR from the OEM's pools. The MO's CA validates the $\mathrm{CSR}$ and generates the corresponding contract certificate Cert $C C$.
Afterwards, the MO's ISO 15118-20 handler encrypts the signature of Cert $_{C C}$ (using $S R K_{\text {pub }}$ and Pol from Cert ${ }_{P C}$ as described in Section 6.4.2). Whenever an MO has read a CSR from the OEM CSR Pool, it is deleted from the pool, since any key pair should only be used for a single certificate.

The MO builds a contract certificate package as per [VDE 2019], where a contract certificate with encrypted signature replaces a plain-text contract certificate and the credential activation information - encrypted private key. The MO sends this contract certificate package to the CPS. The CPS saves the contract certificate packages of all MOs under the PCID in the contract certificate pool.

6.5.3 Contract credential installation. In an ISO 15118-20 charging session, the EVCC sends a CertInstallReq (as described in Section 6.3.2) to the SECC, from where the request is forwarded to the CPS via the CPO. The CPS downloads all contract certificate packages of the EVCC from the Contract Certificate Pool. If there are certificates available, it ignores the CSR from the CertInstallReq and instead uses the pool data. It writes them to the CertInstallRes messages (one per certificate), signs and sends this response to the EVCC via CPO and SECC. The CertInstallReq CSRs can be ignored by the CPS because the certificate pool approach uses an out-of-band mechanism to transfer CSRs to the MO (Section 6.5.2).

The $E_{n c_{S K}}\left(\mathrm{Cert}_{C C}\right)$ in the CertInstallRes does not match the CSR in the CertInstallReq and the EVCC has to use Subject Key Identifier extension of the $E n c_{S K}\left(\mathrm{Cert}_{C C}\right)^{4}$ in order to match the certificate to one of its contract key pairs.

Finally, the EVCC receives the CertInstallRes from the SECC, decrypts the signature of the new contract certificate as described in Section 6.4.2, and can use it in accordance with Section 6.4.3.

6.5.4 Uploading new CSRs into the pool. Since used CSRs are deleted from the OEM CSR Pool, new CSRs might have to be uploaded in the future. For this, the EVCC can use its TPM to generate new contract key pairs with corresponding CSRs at any time and send the CSRs to its OEM via the vehicle's telematics system. Note that telematics systems are commonly available in modern vehicles;

\footnotetext{
${ }^{4}$ Note that ISO 15118-20 requires all contract certificates to contain the Subject Key Identifier extension in accordance with [Cooper et al. 2008], Section 4.2.1.2, Method (2) and that the Subject Key Identifier is in plaintext since only the signature of $\mathrm{Cert}_{C C}$ is encrypted.
} 
hence, this requirement is not limiting. For example, Ford Motor Co. announced that they are planning to use a telematics-based method to provision ISO 15118 contract credentials [Berman 2020].

\section{EVALUATION}

To evaluate HIP-20, we discuss how the system requirements defined in Section 5 are met. The security requirements are discussed in Section 7.1 and the functional requirements in Section 7.2.

\subsection{Security Discussion}

The security of HIP-20 is based on the security of the vehicle's TPM as the security anchor. Thus, a TPM must be used which has been systematically designed, reviewed, and tested to fulfill certain security and safety requirements. In the following, we discuss how HIP addresses the security requirements defined in Section 5.

$R S_{1}$ Secure key generation: OEM provisioning key pair $P C$ and contract credential key pair $C C$ are generated by the TPM (using the TPM's random number generator) and stored "under" the $S R K$ (cf. Sections 6.4.1 and 6.4.2). Assuming the key generation of the used TPM is implemented correctly, $R S_{1}$ is met.

$R S_{2}$ Secure key storage: As described in Section 6.3.1, PC and $C C$ (and also $S R K$ ) carry the attributes fixedTPM and fixedParent preventing the export of private keys. Thus, only $P C_{p u b}$ and $C C_{p u b}$ can be exported and transmitted to other parties, e.g., backend systems. The private keys are securely stored in the TPM's shielded location. Thus, neither a backend hacker nor a car hacker can read out private keys and security requirement $R S_{2}$ is fulfilled.

$R S_{3}$ Secure cryptographic operations: $R S_{3}$ is also fulfilled, since the critical private keys are only used in the shielded area of the TPM and cannot be exported.

$R S_{4}$ Key usage authorization: $R S_{4}$ is met because the TPM checks the security policy every time a private key (OEM provisioning private key or contract certificate private keys) is accessed. By setting a policy TPM2_PolicyPCR (cf. Section 6.3.1), access to keys is only possible if the EVCC is in a trustworthy state.

$R S_{5}$ Cryptographic agility: The TPM 2.0 is specified with a cryptographically agile interface. Modern TPMs already support multiple algorithms without any changes to the interaction model. Key lengths and algorithms can be chosen by simply altering the input structure. HIP works independently of the selected cryptographic algorithms. Insecure cryptographic algorithms can be easily exchanged. In rolled out systems, this would either require updating the firmware of the TPM or, if this is not possible, replacing the TPM. Security requirement $R S_{5}$ is fulfilled.

$R S_{6}$ Trustworthy credential enrollment: The EVCC can only decrypt the signature of $\mathrm{Cert}_{C C}$ if the corresponding contract key was generated in the same TPM as the SRK and sealed to the defined policy (cf. certificate TPM extension in Fig. 3) and also has the expected attributes (cf. Table 1). For a successful attack, an adversary would need to compromise the MO backend and to illegitimately control the TPM (e.g., by a run-time attack) to en-/decrypt the Cert $_{C C}$ signature (e.g., with non-trustworthy parameters). This attack vector is more complicated than a direct $\mathrm{MO}$ backend misuse and thus beyond our adversary model. Security requirement $R S_{6}$ is fulfilled.

\subsection{Discussion of Functional Requirements}

For the functional evaluation, we enhanced the implementation from HIP [Fuchs et al. 2020a] with the relevant changes for HIP20. We used an automotive qualified and Common Criteria EAL4+ certified Infineon OPTIGA TPM SLI 9670 [Infineon 2019] for performance measurements.

$R F_{1}$ Minimal overhead: HIP-20 creates overhead in the credential provisioning process of ISO $15118-20$. To show $R F_{1}$ is fulfilled, we verify that that this overhead stays within the standard's limits.

With respect to the communication overhead, the $\operatorname{Cert}_{P C}$ using the values from Fig. 3 was 676 bytes (increase of 171 bytes due to the new extension). The $\operatorname{Cert}_{C C}$ with an encrypted signature was 538 bytes (increase of 32 byte due to IV and MAC tag). Hence, both certificates stayed within the 800 byte limit of ISO 15118-20. For HIP20 , the encrypted session key $S K$ is transmitted in the contract key field of CertInstallRes and requires 50 bytes, ${ }^{5}$ staying within the ISO 15118-20 limit of 64 bytes. The CSR (198 bytes) uses a new field and is thus not affected by current limits. The total communication overhead is with 369 bytes in certificate installation requests and 18 bytes in the respective responses relatively low.

Regarding computational overhead, the only changes are to MO and EVCC. Note that with the DKE application guideline the MO's processes do not affect the ISO 15118-20 protocol flow. Thus, the only relevant overhead is at the EVCC due to the added TPM interactions for key generation, signatures (with policy checks), and decryption of the contract certificate's signature (based on TPM2_ActivateCredential ${ }^{6}$ ). We measured the respective times in isolation, i.e., without ISO 15118-20 communication. All measurements were repeated 100 times resulting in the following averages: $216.4 \mathrm{~ms}$ for the generation of contract keys, $552.1 \mathrm{~ms}$ for signatures, ${ }^{7}$ and 230.9 ms for the TPM2_ActivateCredential.

Note that it is possible to complete the contract key generation as well as the signatures of CertInstallReq and CSR independently of the ISO 15118 communication session, as none of these actions require session specific information. Additionally, [Fuchs et al. 2020b] shows that it is possible to pre-calculate all TPM policies for timecritical signatures in ISO 15118-20, resulting in a time for signature generation of only $228.8 \mathrm{~ms}$. Hence, the computational overhead of HIP-20 is limited to $t_{\text {auth }}=228.8 \mathrm{~ms}-t_{\text {sig }}$ during EVCC authentication (whereby $t_{s i g}$ is the time for signature generation on the EVCC) and $t_{d e c}=230.9 \mathrm{~ms}-t_{e c d h}$ during the credential provisioning process (with $t_{e c d h}$ being the time for ECDH-based key generation on the EVCC). Assuming the performance times of an automotive controller as reported in [Popa et al. 2019], ${ }^{8}$ (i.e., $t_{s i g}=46.3 m s$ and $t_{e c d h}=59.8 \mathrm{~ms}$ ) the overhead of HIP-20 is $t_{\text {auth }}=182.5 \mathrm{~ms}$ and $t_{\text {dec }}=171.1 \mathrm{~ms}$.

$R F_{2}$ ISO 15118-20 conformance: HIP-20 alters the ISO 15118-20 protocol slightly to increase security. The CertInstallReq message is changed and a minor protocol version number is introduced. We also redefined the fields in the response. The message flow remained

\footnotetext{
${ }^{5} 32$ byte HMAC and 18 byte encrypted ( $\left.S K_{\text {size }} \| S K\right)$, required by the TPM. ${ }^{6} \mathrm{We}$ assume that the difference between the symmetric decryption of the contract certificate's signature in HIP-20 and the symmetric decryption of $C C_{p r}$ in ISO 15118-20 is negligible, i.e., the only difference in the credential provisioning process is between TPM2_ActivateCredential and the usual ECDH (both resulting in a symmetric key).

${ }^{7}$ The policy was a TPM2_PolicyAuthorize, used to authorize a TPM2_PolicyPCR.

${ }^{8}$ A 32 bit Infineon TC297 with the wolfCrypt library and the secp256r1 curve.
} 
unchanged. As these minimal changes are required to substantially increase the security, we argue requirement $R F_{2}$ is met.

$R F_{3}$ Backwards compatibility: Since HIP-20 does not change the general ISO 15118 message flow and the introduced certificate extension is marked as non-critical, intermediate nodes (e.g., the SECC) simply forward the messages ensuring backwards compatibility. Thus, requirement $R F_{3}$ is fulfilled.

$R F_{4}$ Conformance to DKE application guideline: HIP-20 requires minimal changes for integrating the TPM but the general certificate installation process described in the application guideline is unchanged. Thus, we argue requirement $R F_{4}$ is met.

$R F_{5}$ Conformance to standard CA procedures: HIP-20 integrates CSRs into ISO 15118-20 CertInstallReq messages and makes them compatible with the standard procedures followed by CAs. HIP-20 adds an additional step of credential activation to these procedures ensuring that the issued credential can only be used by the designated vehicle. Thus, HIP-20 provides stronger security guarantees compared to the common practice. We consider $R F_{5}$ is fulfilled.

\section{CONCLUSION}

In this paper, we describe a comprehensive solution for Plug-andCharge that strongly reduces the risks of account breaches for emobility users to adversaries targeting vehicles or backend systems. The solution moves the generation of sensitive contract credentials out of the backend systems of the service providers to the vehicle's TPM and defines a credential activation process compatible with the ISO 15118-20 protocol flow, application guideline VDE-AR-E 2802100-1 and the standard procedures of the involved CAs. In contrast to these existing credential provisioning processes, HIP-20 offers the service provider strong security guarantees that the credential is indeed used by the intended EV, due to the certified features of the TPM 2.0. The respective proposal for change enabling HIP-20 support in Plug-and-Charge has been submitted to the ISO 15118-20 Security Task Force and is currently under consideration.

\section{ACKNOWLEDGMENTS}

The work presented in this paper has been partly funded by the German Federal Ministry for Economic Affairs and Energy under the project "LamA-connect", by the German Federal Ministry of Education and Research and the Hessian Ministry of Higher Education, Research, Science and the Arts within their joint support of the National Research Center for Applied Cybersecurity ATHENE, and the TALENTA program of the Fraunhofer-Gesellschaft.

\section{REFERENCES}

AUTOSAR. 2017. Specification of Secure Onboard Communication https://www.autosar.org/fileadmin/user_upload/standards/classic/43/AUTOSAR SWS SecureOnboardCommunication.pdf.

B. Berman. 2020. The ISO standard for electric-vehicle "Plug-and-Charge" faces security concerns. https://www.sae.org/news/2020/08/iso-ev-plug-and-charge-standardfaces-security-concerns Retrieved on 04.09.2020.

L. Buschlinger, M. Springer, and M. Zhdanova. 2019. Plug-and-Patch: Secure Value Added Services for Electric Vehicle Charging. In Proceedings of the 14th International Conference on Availability, Reliability and Security, ARES 2019, Canterbury, UK, August 26-29, 2019. 2:1-2:10.

CAR 2 CAR Communication Consortium. 2018. Protection Profile V2X Hardware Security Module, Release 1.3.0.

S. Checkoway, D. McCoy, B. Kantor, D. Anderson, H. Shacham, S. Savage, K. Koscher A. Czeskis, F. Roesner, and T. Kohno. 2011. Comprehensive Experimental Analyses of Automotive Attack Surfaces. In Proceedings of the 20th USENIX Conference on Security (SEC'11). USENIX Association, Berkeley, CA, USA, 6-6.
D. Cooper, S. Santesson, S. Farrell, S. Boeyen, R. Housley, and W. Polk. 2008. Internet X.509 Public Key Infrastructure Certificate and Certificate Revocation List (CRL) Profile. RFC 5280. RFC Editor. http://www.rfc-editor.org/rfc/rfc5280.txt

J. Van den Herrewegen and F. Garcia. 2018. Beneath the bonnet: A breakdown of diagnostic security. In European Symposium on Research in Computer Security. Springer, 305-324.

D. Dolev and A. Yao. 1983. On the security of public key protocols. IEEE Transactions on Information Theory 29, 2 (Mar 1983), 198-208. https://doi.org/10.1109/TIT.1983. 1056650

A. Fuchs, D. Kern, C. Krauß, and M. Zhdanova. 2020a. HIP: HSM-based Identities for Plug-and-Charge. In Proceedings of the 15th International Conference on Availability, Reliability and Security, ARES. ACM. https://doi.org/10.1145/3407023.3407066

A. Fuchs, D. Kern, C. Krauß, and M. Zhdanova. 2020b. Securing Electric Vehicle Charging Systems Through Component Binding. In International Conference on Computer Safety, Reliability, and Security. Springer, 387-401.

A. Fuchs, D. Kern, C. Krauß, and M. Zhdanova. 2020c. TrustEV: Trustworthy Electric Vehicle Charging and Billing. In Proceedings of the 35th ACM/SIGAPP Symposium on Applied Computing SAC 2020. ACM.

A. Fuchs, C. Krauß, and J. Repp. 2016. Advanced Remote Firmware Upgrades Using TPM 2.0. In ICT Systems Security and Privacy Protection. Springer International Publishing, Cham, 276-289.

D. Ghosh, R. Thomas, and S. Wicker. 2013. A privacy-aware design for the vehicle-togrid framework. In 2013 46th Hawaii International Conference on System Sciences. IEEE, 2283-2291

A. Greenberg, C. Miller, and C. Valasek. 2015. Hackers Remotely Kill a Jeep on the Highway - With Me in It.

HIS. 2009. SHE - Secure Hardware Extension Functional Specification.

Hubject GmbH. 2020. Hubject Plug\&Charge Certificate Policy (CP) for the Hubject ISO 15118 V2G PKI. Version 1.7.

Infineon. 2019. OPTIGATM TPM SLI 9670. https://www.infineon.com/cms/en/product/ security-smart-card-solutions/optiga-embedded-security-solutions/optigatpm/sli-9670/. Retrieved on 01.04.2020.

ISO/IEC. 2013. Road vehicles - Vehicle to grid communication interface - Part 1: General information and use-case definition. ISO Standard 15118-1:2013.

ISO/IEC. 2014. Road vehicles - Vehicle-to-Grid Communication Interface - Part 2: Network and application protocol requirements. ISO Standard 15118-2:2014.

ISO/IEC. 2019. Road vehicles - Vehicle-to-Grid Communication Interface - Part 20: 2nd generation network and application protocol requirements. ISO/preFDIS 15118-20.

K. Koscher, A. Czeskis, F. Roesner, S. Patel, T. Kohno, S. Checkoway, D. McCoy, B. Kantor, D. Anderson, H. Shacham, and S. Savage. 2010. Experimental Security Analysis of a Modern Automobile. In 2010 IEEE Symposium on Security and Privacy. 447-462.

C. Miller and C. Valasek. 2014a. Adventures in Automotive Networks and Control Units.

C. Miller and C. Valasek. 2014b. A Survey of Remote Automotive Attack Surfaces. In Blackhat.

M. Nystrom and B. Kaliski. 2000. PKCS \#10: Certification Request Syntax Specification Version 1.7. RFC 2986. RFC Editor. http://www.rfc-editor.org/rfc/rfc2986.txt

L. Popa, B. Groza, and P. Murvay. 2019. Performance evaluation of elliptic curve libraries on automotive-grade microcontrollers. In Proceedings of the 14th International Conference on Availability, Reliability and Security. 1-7.

I. Rouf, R. Miller, H. Mustafa, T. Taylor, S. Oh, W. Xu, M. Gruteser, W. Trappe, and I. Seskar. 2010. Security and Privacy Vulnerabilities of In-car Wireless Networks: A Tire Pressure Monitoring System Case Study. In Proceedings of the 19th USENIX Conference on Security. USENIX Association, Berkeley, CA, USA, 21-21.

N. Saxena, S. Grijalva, V. Chukwuka, and A. Vasilakos. 2017. Network security and privacy challenges in smart vehicle-to-grid. IEEE Wireless Communications 24, 4 (2017), 88-98.

Trusted Computing Group 2014. Trusted Platform Module Library Specification (Family 2.0, Level 00, Revision 01.16 ed.). Trusted Computing Group.

Trusted Computing Group. 2016. Trusted Platform Module Library - Part 1: Architecture. Specification Family 2.0 - Rev. 01.38.

Trusted Computing Group. 2017. TCG TPM v2.0 Provisioning Guidance. Guidance Ver. 1.0 - Rev. 1.0.

VDE. 2019. Handling of certificates for electric vehicles, charging infrastructure and backend systems within the framework of ISO 15118. VDE-AR-E 2802-100-1:2019-12.

C. Xu, H. Liu, P. Li, and P. Wang. 2018. A Remote Attestation Security Model Based on Privacy-Preserving Blockchain for V2X. IEEE Access 6 (2018), 67809-67818.

D. Zelle, C. Krauß, H. Strauß, and K. Schmidt. 2017. On Using TLS to Secure InVehicle Networks. In Proceedings of the 12th International Conference on Availability, Reliability and Security (ARES).

D. Zelle, M. Springer, M. Zhdanova, and C. Krauß. 2018. Anonymous Charging and Billing of Electric Vehicles. In Proceedings of the 13th International Conference on Availability, Reliability and Security, ARES. ACM, 22:1-22:10.

T. Zhao, C. Zhang, L. Wei, and Y. Zhang. 2015. A secure and privacy-preserving payment system for Electric vehicles. In Communications (ICC), 2015 IEEE International Conference on. IEEE, 7280-7285. 